\title{
Onset of convection for autocatalytic reaction fronts in a vertical slab
}

\author{
Jie Huang, Desiderio A. Vasquez, ${ }^{*}$ and Boyd F. Edwards \\ Department of Physics, West Virginia University, P.O. Box 6315, Morgantown, West Virginia 26506-6315 \\ Paul Kolodner \\ AT\&T Bell Laboratories, Murray Hill, New Jersey 07974-0636
}

(Received 19 July 1993)

\begin{abstract}
A fully three-dimensional linear stability analysis shows that ascending autocatalytic reaction fronts in vertical slabs are unstable to convection for large-wavelength perturbations at all finite values of the dimensionless driving parameter $S=\delta g a^{3} / v D_{C}$. This parameter involves a fractional density difference $\delta$ between the unreacted and reacted fluids, the acceleration of gravity $g$, the slab width $a$, the kinematic viscosity $v$, and the catalyst molecular diffusivity $D_{C}$. Buoyancy dominates over the competing curvature dependence of the front velocity in a band $0<q<q_{c}$ of unstable dimensionless wave numbers, with $q_{c} \rightarrow S / 24$ as $S \rightarrow 0$ and $q_{c} \rightarrow(S / 4)^{1 / 3}$ as $S \rightarrow \infty$. As $S \rightarrow 0$, the perturbation with wave number $q_{m}=q_{c} / 2$ has the maximum dimensionless growth rate $\sigma_{m}=D_{C} S^{2} / 48 v$. For general $S$, the cutoff wave number $q_{c}$ is calculated using exact analytical solutions for the perturbed fluid velocity. The calculated results should be observable in experiments.
\end{abstract}

PACS number(s): 47.20.Bp, 47.70.Fw, 03.40.Gc

\section{INTRODUCTION}

Recent experiments observe steady axisymmetric convection near iodate-arsenous acid reaction fronts in vertical capillary tubes $[1,2]$. This convection is driven by the buoyancy of the lighter reacted fluid below the ascending front; descending fronts involve no convection. Descending fronts are flat and propagate at a fixed speed which is independent of the tube diameter. When the tube diameter is less than a critical diameter $d_{c}$ of about $1 \mathrm{~mm}$, the upward propagation speed and the corresponding curvature of the front are the same as for the descending front, indicating the absence of convection. However, when the tube diameter is raised above $d_{c}$, the upward propagation speed increases and the curvature of the front becomes nonzero, indicating the onset of convection. In these experiments, the tube diameter strongly restricts the possible wavelengths of the front perturbations. In contrast, for a vertical slab, the horizontal freedom parallel to the slab allows unrestricted perturbation wavelengths in this direction. Thus, experiments in this geometry might be expected to reveal rich information about wavelength selection in chemical waves. The purpose of this paper is to anticipate such experiments by studying the linear stability of three-dimensional flows in a vertical slab.

The reaction kinetics of a propagating front of iodide in iodate-arsenous acid solution can be accurately described by the oxidation of iodide by iodate and the reduction of iodine by arsenous acid [3]. The iodide is generated autocatalytically at the reaction front and

\footnotetext{
*Present address: Department of Physics, Indiana University-Perdue University at Fort Wayne, Fort Wayne, IN 46805-1499.
}

diffuses ahead of the front. The coupling of the corresponding chemical reaction-rate equations with the catalyst diffusion equation governs the spatiotemporal evolution of the iodide concentration and the propagation speed of the iodide front. A simple autocatalytic reaction-diffusion equation was derived from these equations. In the absence of convection, this equation successfully describes one dimensional propagation of the iodide front in a vertical capillary tube. This front is very thin and is flat in the tube. The calculated propagation speed of the front is in good agreement with experiments [3].

A hydrodynamic stability theory of convection near autocatalytic reaction fronts [4] treats the thin chemical reaction front as a moving surface which consumes unreacted fluid of uniform mass density $\rho_{u}$ to produce reacted fluid of lower uniform density $\rho_{r}$, thereby relegating all chemical reactions to the surface. In this "thinfront" approximation, the reaction-diffusion equation reduces to a simple "eikonal" relation [5] between the front velocity and the front curvature. In a reference frame stationary with respect to the fluid, the eikonal relation gives the normal component of velocity of the reaction front $c=c_{0}+D_{C} K$, where $c_{0}$ is the flat front speed, $D_{C}$ the molecular diffusivity of the catalyst, and $K$ the front curvature. Here $K$ is measured as positive when the center of curvature is in the unreacted fluid. This curvature correction tends to lower peaks and to raise valleys in the front surface, thus flattening the front. For ascending fronts, buoyancy competes with this curvature effect and tends to destabilize a flat front in favor of convection. The relative strength of buoyancy is measured by a dimensionless driving parameter appropriate for vertical slabs and cylinders (Ref. [6]),

$$
S=\frac{\delta g a^{3}}{v D_{C}}
$$


This parameter involves a fractional density difference $\delta=\left(\rho_{u}-\rho_{r}\right) / \rho_{r}$, the acceleration of gravity $g$, the slab width or cylinder radius $a$, and the kinematic viscosity $v$. The small chemical concentrations typical of experiments imply small $\delta \approx 10^{-4}$ and negligible differences in $v$ and $D_{C}$ between the reacted and unreacted fluids. The density difference $\delta$ can be due to differences of both temperature and chemical composition. However, the thermal difference can be neglected because the thermal diffusivity is effectively infinite [7]. Linear stability analyses in the thin-front approximation performed for twodimensional flows [6] in vertical slabs and in vertical cylinders show that convection occurs only above a finite critical value $S_{c}$, where curvature effects and buoyancy exactly balance each other and the system is marginally stable. The predicted critical diameter for onset of convection agrees with the observed critical diameter for ascending reaction fronts in vertical capillary tubes filled with iodate-arsenous acid solutions. Furthermore, these results obtained using the thin-front approximation agree with results based on the autocatalytic reaction-diffusion equation [8].

In this paper, a linear stability analysis of threedimensional flows in vertical slabs using the NavierStokes equations and the thin-front approximation shows that the freedom afforded by horizontal motion parallel to the slab, neglected in the previous two-dimensional calculations, renders $S_{c}=0$ in this geometry. For finite $S$, perturbations with wave numbers satisfying $0<q<q_{c}$, where $q_{c}$ is a cutoff wave number, are unstable to convection, with $q_{c} \rightarrow 0$ as $S \rightarrow 0$. A relation obtained between $S$ and $q_{c}$ is universal, being independent of the slab width and any fluid parameters, and therefore applies generally to experiments. In Sec. II, we outline the basic equations, boundary conditions, and reaction front jump conditions. We obtain the general analytical solutions of threedimensional flows in vertical slabs in Sec. III. For general $S$, both approximate and exact results for the marginal state are given and discussed in Sec. IV. In Sec. V, we study the limit of $S \rightarrow 0$, the Hele-Shaw limit, in which the Navier-Stokes equations are replaced by Darcy's law. In Sec. VI, we discuss the laterally unbounded $(S \rightarrow \infty)$ limit, construct a useful approximate analytical result for the marginal state, draw conclusions, and discuss implications for experiments.

\section{EQUATIONS OF MOTION}

The fluid velocity $\mathbf{v}(\mathbf{x}, t)$ and the reaction front height $z=h(x, y, t)$ evolve according to the dynamical equations [4]

$$
\begin{aligned}
& \frac{\partial \mathbf{v}}{\partial t}+(\mathbf{v} \cdot \nabla) \mathbf{v}=-\rho \hat{\mathbf{z}}-\nabla P+\nabla^{2} \mathbf{v}, \\
& \boldsymbol{\nabla} \cdot \mathbf{v}=0, \\
& \widehat{\mathbf{n}} \cdot\left[\widehat{\mathbf{z}} \frac{\partial h}{\partial t}-\left.\mathbf{v}\right|_{z=h}\right]=v_{0}+\frac{D_{C}}{v} \kappa,
\end{aligned}
$$

where length, time, pressure, and density are measured in units of $a, a^{2} / v, v^{2} \rho_{r} / a^{2}$, and $\widetilde{\rho}=v^{2} \rho_{r} / g a^{3}$, respectively, and where we have neglected the small density difference between the reacted and unreacted fluids by setting $\rho=\rho_{r} / \widetilde{\rho}$ everywhere except in the large gravity term [the first term on the right side of Eq. (2a)], consistent with the Oberbeck-Boussinesq approximation [9]. The first two equations express conservation of momentum and mass. The third is the dimensionless eikonal relation, which involves a dimensionless flat-front speed $v_{0}=c_{0} a / v$ and a dimensionless curvature $\kappa$. Again neglecting the density difference, the matching and boundary conditions include continuous fluid velocity $\mathbf{v}$ and continuous stress $n_{j} T_{i j}$ at the front as well as vanishing velocity at the no-slip impenetrable sidewalls. Here, the $n_{j}$ are the cartesian components of the normal vector $\hat{\mathbf{n}}$ pointing into the unreacted fluid and $T_{i j}=P \delta_{i j}-\partial v_{i} / \partial x_{j}-\partial v_{j} / \partial x_{i}$ is the dimensionless stress tensor.

To study the onset of convection, it is convenient to introduce a reduced pressure $p=P+\rho z$ and to allow small perturbations about an ascending convectionless flat front described in the comoving frame by $\mathbf{v}^{(0)}=-v_{0} \widehat{\mathbf{z}}, h^{(0)}=0$, $\widehat{\mathbf{n}}^{(0)}=\widehat{\mathbf{z}}$, and $\boldsymbol{\kappa}^{(0)}=0$. Linearizing in the perturbations yields

$$
\begin{aligned}
& \frac{\partial \mathbf{v}^{(1)}}{\partial t}-v_{0} \frac{\partial \mathbf{v}^{(1)}}{\partial z}=-\nabla p^{(1)}+\nabla^{2} \mathbf{v}^{(1)} \quad(z \neq 0), \\
& \nabla \cdot \mathbf{v}^{(1)}=0, \\
& \frac{\partial h^{(1)}}{\partial t}=\frac{D_{C}}{v} \nabla^{2} h^{(1)}+\left.\widehat{\mathbf{z}} \cdot \mathbf{v}^{(1)}\right|_{z=0},
\end{aligned}
$$

where $\hat{\mathbf{n}}^{(1)}=-\nabla h^{(1)}$ and $\kappa^{(1)}=\nabla^{2} h^{(1)}$. Continuous fluid velocity $\mathbf{v}$, tangential stress $\epsilon_{i j k} n_{j} n_{l} T_{k l}$, and normal stress $n_{i} n_{j} T_{i j}$ at the front and the continuity equation require

$$
\begin{aligned}
& {\left[\mathbf{v}^{(1)}\right]_{-}^{+}=0,} \\
& {\left[\frac{\partial}{\partial z} \mathbf{v}^{(1)}\right]_{-}^{+}=0,} \\
& {\left[p^{(1)}\right]_{-}^{+}=\frac{D_{C}}{v} S h^{(1)},}
\end{aligned}
$$

to first order in the perturbations. Here, $[q]_{-}^{+}$ $=\lim _{\epsilon \rightarrow 0}\left(\left.q\right|_{z=+\epsilon}-\left.q\right|_{z=-\epsilon}\right)$ is the difference between the values of a quantity $q$ above and below the front, which is located at $z=0$ for terms already first order in the perturbations.

To state the final form of the equations to be solved, we first define the coordinate system of the vertical slab by $|x| \leq \frac{1}{2}$ in dimensionless units, with $\hat{\mathbf{z}}$ up. For the marginal state, the perturbations neither grow nor decay with time so that $\partial / \partial t=0$. Setting $\mathbf{v}^{(1)}=(u, v, w)$ and taking the curl of Eq. (3a), we have

$$
\begin{aligned}
& \nabla^{2} \frac{\partial w}{\partial y}-\nabla^{2} \frac{\partial v}{\partial z}=0 \\
& \nabla^{2} \frac{\partial u}{\partial z}-\nabla^{2} \frac{\partial w}{\partial x}=0 \\
& \nabla^{2} \frac{\partial v}{\partial x}-\nabla^{2} \frac{\partial u}{\partial y}=0
\end{aligned}
$$


The second term in Eq. (3a) leads to corrections in $S_{c}$ of relative order $10^{-5}$ for two-dimensional flows in vertical slabs and in vertical cylinders [6]. We expect that this term leads to corrections of the same order for threedimensional flows in vertical slabs. Thus, this term is neglected. The vanishing velocity at the no-slip impenetrable sidewalls requires the six independent boundary conditions

$\left.u\right|_{x= \pm 1 / 2}=0,\left.\quad v\right|_{x= \pm 1 / 2}=0$, and $\left.w\right|_{x= \pm 1 / 2}=0$.

Equations (3) yield the six independent matching conditions

$$
\begin{aligned}
& {[v]_{-}^{+}=0, \quad\left[\frac{\partial v}{\partial z}\right]_{-}^{+}=0, \quad[w]_{-}^{+}=0,} \\
& {\left[\frac{\partial w}{\partial z}\right]_{-}^{+}=0,\left[\frac{\partial^{2} w}{\partial z^{2}}\right]_{-}^{+}=0,} \\
& \text { and }\left[\frac{\partial^{3} w}{\partial z^{3}}\right]_{-}^{+}=\left.S w\right|_{z=0} .
\end{aligned}
$$

\section{ANALYTICAL SOLUTIONS}

The unboundedness in the $y$ direction allows the perturbation wavelength to be chosen freely in this direction, so we write $\mathbf{v}^{(1)}$ as

$$
\begin{aligned}
& u(x, y, z)=\widetilde{u}(x) e^{k z} \sin q y, \\
& v(x, y, z)=\widetilde{v}(x) e^{k z} \cos q y, \\
& w(x, y, z)=\widetilde{w}(x) e^{k z} \sin q y .
\end{aligned}
$$

Substituting Eqs. (5) into Eqs. (4) gives two independent ordinary differential equations

$$
\begin{aligned}
& q\left(\frac{d^{2}}{d x^{2}}+\beta^{2}\right) \widetilde{w}-k\left(\frac{d^{2}}{d x^{2}}+\beta^{2}\right) \widetilde{v}=0, \\
& k\left(\frac{d^{2}}{d x^{2}}+\beta^{2}\right) \widetilde{u}-\frac{d}{d x}\left[\frac{d^{2}}{d x^{2}}+\beta^{2}\right) \widetilde{w}=0,
\end{aligned}
$$

where $\beta^{2}=k^{2}-q^{2}$. The continuity equation (3b) yields

$$
\frac{d \widetilde{u}}{d x}-q \widetilde{v}+k \widetilde{w}=0 .
$$

Equations (6) are invariant under even reflection of the velocity vector about the $x=0$ plane; $\widetilde{u}(-x)=-\widetilde{u}(x)$, $\widetilde{v}(-x)=\widetilde{v}(x)$, and $\widetilde{w}(-x)=\widetilde{w}(x)$. They are also invariant under odd reflection; $\widetilde{u}(-x)=\widetilde{u}(x), \widetilde{v}(-x)=-\widetilde{v}(x)$, and $\widetilde{w}(-x)=-\widetilde{w}(x)$. These properties will allow us to study even and odd solutions separately for this linear problem.

Equations (6) have solutions for both real and complex $k$. We first prove that $\widetilde{u}=0$ if $k$ is real. Simplifying Eqs. (6) yields

$$
\left(\frac{d^{2}}{d x^{2}}+\beta^{2}\right)^{2} \widetilde{u}=0 .
$$

When $\beta^{2}=0$, the general solution of Eq. (7) is

$$
\widetilde{u}=A_{1}+A_{2} x+A_{3} x^{2}+A_{4} x^{3} .
$$

The boundary conditions $\widetilde{u}\left( \pm \frac{1}{2}\right)=\widetilde{u}^{\prime}\left( \pm \frac{1}{2}\right)=0$ lead to a set of four homogeneous linear algebraic equations for the coefficients, which yields only the vanishing solution $\widetilde{u}=0$. When $\beta^{2} \neq 0$, the general solution of Eq. (7) is

$$
\widetilde{u}=A_{1} \cos \beta x+A_{2} \sin \beta x+A_{3} x \cos \beta x+A_{4} x \sin \beta x .
$$

This solution is valid for both real $\beta\left(k^{2}>q^{2}\right)$ and imaginary $\beta \quad\left(k^{2}<q^{2}\right)$. The boundary conditions $\widetilde{u}\left( \pm \frac{1}{2}\right)=\widetilde{u}^{\prime}\left( \pm \frac{1}{2}\right)=0$ yield a set of four homogeneous linear algebraic equations for the coefficients. For a nonvanishing solution of these equations, the determinant of the coefficient matrix must vanish. This leads to the condition

$$
\sin ^{2} \beta=\beta^{2} .
$$

Equation (1) has no solution for $\beta^{2} \neq 0$, hence $\tilde{u}=0$ if $k$ is real. Since $\widetilde{u}=0$, the corresponding motion of the fluid is restricted to the $y-z$ plane parallel to the slab, and Eqs. (6) reduce to

$$
\widetilde{v}=\frac{q}{k} \widetilde{w}
$$

and

$$
\begin{aligned}
& \frac{d^{3} \widetilde{w}}{d x^{3}}=0, \quad\left(\beta^{2}=0\right), \\
& {\left[\frac{d^{2}}{d x^{2}}+\beta^{2}\right) \widetilde{w}=0, \quad\left(\beta^{2} \neq 0\right) .}
\end{aligned}
$$

Subject to the boundary conditions $\widetilde{w}\left( \pm \frac{1}{2}\right)=0$, we have the general even solutions

$$
\widetilde{w}_{e}=\left\{\begin{array}{l}
A\left(x^{2}-\frac{1}{4}\right), \quad \beta=0 ; \\
A_{n} \cos (2 n+1) \pi x, \quad \beta=(2 n+1) \pi,
\end{array}\right.
$$

and the general odd solutions

$$
\widetilde{w}_{0}=B_{n} \sin (2 n+2) \pi x, \quad \beta=(2 n+2) \pi,
$$

where $A, A_{n}$, and $B_{n}$ are arbitrary coefficients, and $n=0,1,2, \ldots$.

We now look for solutions for complex $k$. It is convenient to define

$$
\gamma^{2}=q^{2}-k^{2}=-\beta^{2} .
$$

Simplifying Eqs. (6), we have

$$
\left(\frac{d^{2}}{d x^{2}}-\gamma^{2}\right)^{2} \widetilde{u}=0,
$$

whose general solution is

$$
\widetilde{u}=E_{1} e^{\gamma x}+E_{2} e^{-\gamma x}+E_{3} x e^{\gamma x}+E_{4} x e^{-\gamma x} .
$$

The boundary conditions $\widetilde{u}\left( \pm \frac{1}{2}\right)=\widetilde{u}^{\prime}\left( \pm \frac{1}{2}\right)=0$ require 


$$
\begin{aligned}
& {\left[\begin{array}{cccc}
e^{-\gamma / 2} & e^{\gamma / 2} & -\frac{1}{2} e^{-\gamma / 2} & -\frac{1}{2} e^{\gamma / 2} \\
e^{\gamma / 2} & e^{-\gamma / 2} & \frac{1}{2} e^{\gamma / 2} & \frac{1}{2} e^{-\gamma / 2} \\
\gamma e^{-\gamma / 2} & -\gamma e^{\gamma / 2} & \left.1-\frac{\gamma}{2}\right] e^{-\gamma / 2} & \left.1+\frac{\gamma}{2}\right] e^{\gamma / 2} \\
\gamma e^{\gamma / 2} & -\gamma e^{-\gamma / 2} & {\left[1+\frac{\gamma}{2}\right) e^{\gamma / 2}} & \left(1-\frac{\gamma}{2}\right) e^{-\gamma / 2}
\end{array}\right]} \\
& \times\left(\begin{array}{l}
E_{1} \\
E_{2} \\
E_{3} \\
E_{4}
\end{array}\right)=\left(\begin{array}{l}
0 \\
0 \\
0 \\
0
\end{array}\right) \text {. }
\end{aligned}
$$

For a nonvanishing solution, the determinant of the coefficient matrix in Eq. (17) must vanish, yielding

$$
\gamma= \pm \sinh \gamma
$$

A little algebra then yields the corresponding coefficients in Eq. (17),

$$
\begin{aligned}
& E_{2}=-\frac{\sinh \gamma}{\gamma} E_{1}, \quad E_{3}=2 \frac{\gamma-e^{\gamma} \sinh \gamma}{\gamma+e^{\gamma} \sinh \gamma} E_{1}, \\
& \text { and } E_{4}=2 \frac{\sinh \gamma-\gamma e^{\gamma}}{\gamma+e^{\gamma} \sinh \gamma} E_{1} .
\end{aligned}
$$

Finally, for even reflection symmetry, we obtain $\gamma=\sinh \gamma$

$\widetilde{u}_{e}=\sinh \gamma x-2 \tanh \frac{\gamma}{2} x \cosh \gamma x$,

$\widetilde{w}_{e}=\frac{k}{\gamma} \tanh \frac{\gamma}{2}\left[\tanh \frac{\gamma}{2} \cosh \gamma x-2 x \sinh \gamma x\right]$,

$\widetilde{v}_{e}=\frac{q}{k} \widetilde{w}_{e}$
For odd reflection symmetry,

$\gamma=-\sinh \gamma$

$\tilde{u}_{0}=\cosh \gamma x-2 \operatorname{coth} \frac{\gamma}{2} x \sinh \gamma x$,

$\widetilde{w}_{0}=\frac{k}{\gamma} \operatorname{coth} \frac{\gamma}{2}\left[\operatorname{coth} \frac{\gamma}{2} \sinh \gamma x-2 x \cosh \gamma x\right]$,

$\widetilde{v}_{0}=\frac{q}{k} \widetilde{\omega}_{0}$

\section{Setting}

$$
\gamma=\left(\gamma_{R}, \gamma_{I}\right)=\gamma_{R}+\sqrt{-1} \gamma_{I}
$$

reduces Eq. (18) to

$$
\left\{\begin{array}{l}
\gamma_{R}=\sinh \gamma_{R} \cos \gamma_{I} \\
\gamma_{I}=\cosh \gamma_{R} \sin \gamma_{I}
\end{array}\right.
$$

or

$$
\left\{\begin{array}{l}
\gamma_{R}=-\sinh \gamma_{R} \cos \gamma_{I} \\
\gamma_{I}=-\cosh \gamma_{R} \sin \gamma_{I}
\end{array}\right.
$$

Equations (23) are invariant under $\gamma_{R} \rightarrow-\gamma_{R}$ and under $\gamma_{I} \rightarrow-\gamma_{I}$, so that if $\left(\gamma_{R}, \gamma_{I}\right)$ is a solution of Eqs. (23), then $\left(\gamma_{R},-\gamma_{I}\right),\left(-\gamma_{R}, \gamma_{I}\right)$, and $\left(-\gamma_{R},-\gamma_{I}\right)$ are also solutions of Eqs. (23). The first few solutions are (2.2507286116, 4.212392 2305), (2.768678 2830, 7.497 676 2778), (3.103 148 7458, $\quad 10.7125373973)$, (3.352 $2098849,13.8999597140), \ldots$. .

Equations (13a) and (13b) for real $k$ and Eqs. (20) and (21) for complex $k$ are exact analytical solutions of Eqs. (6). Numerical solutions of Eqs. (6) obtained using a shooting method agree with these analytical solutions.

\section{MARGINAL STATE FOR GENERAL $S$}

It is instructive to first obtain an approximate analytical condition for the marginal state. Retaining only the two lowest real values of $k$, the vertical velocity takes the form

$$
\begin{aligned}
& w(x, y, z)=\left[(A-C)\left(x^{2}-\frac{1}{4}\right) e^{-q z}+\left(A_{0}-C_{0}\right) \cos \pi x e^{-k_{1} z}\right] \sin q y, \quad(z \geq 0), \\
& w(x, y, z)=\left[(A+C)\left(x^{2}-\frac{1}{4}\right) e^{q z}+\left(A_{0}+C_{0}\right) \cos \pi x e^{k_{1} z}\right] \sin q y, \quad(z<0),
\end{aligned}
$$

where $k_{1}=\sqrt{\pi^{2}+q^{2}}$ and $A, A_{0}, C$, and $C_{0}$ are four arbitrary coefficients. Since $u(x, y, z)=0$ for real $k$, the continuity equation (3b) eliminates two matching conditions in Eq. (4e). Substituting Eq. (24) into Eq. (4e), multiplying by cos $\pi x$, and integrating over $\left[-\frac{1}{2}, \frac{1}{2}\right]$ produces

$$
\left(\begin{array}{cccc}
8 & -\pi^{3} & 0 & 0 \\
8 q^{2} & -\pi^{3} k_{1}^{2} & 0 & 0 \\
0 & 0 & 8 q & -\pi^{3} k_{1} \\
0 & 0 & 8\left(q^{3}+S / 2\right) & -\pi^{3}\left(k_{1}^{3}+S / 2\right)
\end{array}\right)\left(\begin{array}{l}
C \\
C_{0} \\
A \\
A_{0}
\end{array}\right)=\left(\begin{array}{l}
0 \\
0 \\
0 \\
0
\end{array}\right)
$$


The first two equations yield $C=C_{0}=0$. The nonvanishing solution for the coefficients $A$ and $A_{0}$ requires the determinant of the coefficient matrix in Eq. (25) to vanish, which leads to

$$
S=2 q_{c}\left(\pi^{2}+q_{c}^{2}+q_{c} \sqrt{\pi^{2}+q_{c}^{2}}\right) .
$$

Equation (26) is an approximate condition between the driving parameter $S$ and the cutoff perturbation wave number $q_{c}$ at the marginal state; the ascending flat front is linearly unstable to convection for $q<q_{c}$ at fixed $S$. Since $q_{c} \geq 0$, Eq. (26) cannot be satisfied for $S<0$ (downward propagation of the reaction front). Thus, convection is forbidden for descending fronts. Eq. (26) shows that the cutoff wave number $q_{c}$ of the perturbation is uniquely determined by the driving parameter $S$.

The general even solution of Eqs. (6) for the marginal state can be written as a linear superposition of even solutions with both real and complex $k$ :

$v_{e}(x, y, z)=\left\{-A\left(x^{2}-\frac{1}{4}\right) e^{-q z}-\sum_{n=0}^{\infty} A_{n} \frac{k_{2 n+1}}{q} \cos (2 n+1) \pi x e^{-k_{2 n+1} z}-\sum_{\gamma}\left[A(\gamma) \frac{q}{k_{\gamma}} \widetilde{w}_{e}(x, \gamma) e^{-k_{\gamma} z}+\mathrm{c} . \mathrm{c}.\right]\right\} \cos q y$,

$$
(z \geq 0) \text {, }
$$

$w_{e}(x, y, z)=\left\{A\left(x^{2}-\frac{1}{4}\right) e^{-q z}+\sum_{n=0}^{\infty} A_{n} \cos (2 n+1) \pi x e^{-k_{2 n+1} z}+\sum_{\gamma}\left[A(\gamma) \widetilde{w}_{e}(x, \gamma) e^{-k_{\gamma} z}+\right.\right.$ c.c. $\left.]\right\} \sin q y$

and

$$
\begin{aligned}
& v_{e}(x, y, z)=\left\{C\left(x^{2}-\frac{1}{4}\right) e^{q z}+\sum_{n=0}^{\infty} C_{n} \frac{k_{2 n+1}}{q} \cos (2 n+1) \pi x e^{k_{2 n+1} z}+\sum_{\gamma}\left[C(\gamma) \frac{q}{k_{\gamma}} \widetilde{w}_{e}(x, \gamma) e^{k \gamma^{z}}+\text { c.c. }\right]\right\} \cos q y, \\
& w_{e}(x, y, z)=\left\{C\left(x^{2}-\frac{1}{4}\right) e^{q z}+\sum_{n=0}^{\infty} C_{n} \cos (2 n+1) \pi x e^{k_{2 n+1} z}+\sum_{\gamma}\left[C(\gamma) \widetilde{w}_{e}(x, \gamma) e^{k \gamma^{z}}+\text { c.c. }\right]\right\} \sin q y
\end{aligned}
$$

where

$$
\begin{aligned}
& k_{2 n+1}=\sqrt{(2 n+1)^{2} \pi^{2}+q^{2}}, n=0,1,2, \ldots, \\
& k_{\gamma}=\sqrt{q^{2}-\gamma^{2}} \text { with } \operatorname{Re} k_{\gamma}>0, \\
& \widetilde{w}_{e}(x, \gamma)=\frac{k_{\gamma}}{\gamma} \tanh \frac{\gamma}{2}\left[\tanh \frac{\gamma}{2} \cosh \gamma x-2 x \sinh \gamma x\right] .
\end{aligned}
$$

Here, $A, C, A_{n}, C_{n}, A(\gamma)$, and $C(\gamma)$ are arbitrary coefficients, c.c. denotes the complex conjugate, and we have required the velocity to vanish at $z \rightarrow \pm \infty$. These solutions must satisfy the six independent matching conditions in Eqs. (4e). Accordingly, substituting these solutions into Eqs. $(4 \mathrm{e})$, multiplying by $\cos (2 m+1) \pi x$, and integrating over $\left[-\frac{1}{2}, \frac{1}{2}\right]$ yields an infinite set of linear homogeneous algebraic equations for the coefficients. These equations can be decoupled into two subsets of equations. One subset involves only the combinations $A-C, A_{n}-C_{n}$, and $A(\gamma)-C(\gamma)$, is independent of $S$, and yields the unique solution $A=C, A_{n}=C_{n}$, and $A(\gamma)=C(\gamma)$. The other subset is

$$
\begin{aligned}
& \sum_{\gamma}\left[\frac{\gamma^{2}}{k_{\gamma}} b_{m}(\gamma) A(\gamma)+\text { c.c. }\right]=0, \\
& 2 a_{m} q A+k_{2 m+1} A_{m}+\sum_{\gamma}\left[2 k_{\gamma} b_{m}(\gamma) A(\gamma)+\text { c.c. }\right]=0, \\
& a_{m}\left(2 q^{3}+S\right) A+\left(k_{2 m+1}^{3}+S / 2\right) A_{m}+\sum_{\gamma}\left[\left(2 k_{\gamma}^{3}+S\right) b_{m}(\gamma) A(\gamma)+\text { c.c. }\right]=0,
\end{aligned}
$$

where

$$
a_{m}=4(-1)^{m+1} /(2 m+1)^{3} \pi^{3}
$$

$b_{m}=8(-1)^{m}(2 m+1) \pi k_{\gamma} \sinh \frac{\gamma}{2} /\left[(2 m+1)^{2} \pi^{2}+\gamma^{2}\right]^{2}$, and $m=0,1,2, \ldots$. Requiring the determinant of the coefficient matrix in Eqs. (28) to vanish yields the generalized marginal condition between $S$ and the cutoff wave number $q_{c}$. To determine the numerical relation between $S$ and $q_{c}$, we truncate the infinite expansion in Eqs. (28) to a finite number $N$ of terms. A one-term truncation (a $3 \times 3$ matrix) recovers the approximate analytical relation, Eq. (26). For larger $N$ and a given value of $q_{c}$, we adjust $S$ numerically to make the determinant vanish. To 
test the convergence for increasing $N$, we obtained $S=34.0795,34.1214$, and 34.1351 for the $20-, 30-$, and 40-term truncations for $q_{c}=1.0$, indicating good convergence of these successive truncations. The resulting relation between $S$ and $q_{c}$ for $N=40$ is plotted in Fig. 1. Using the same technique, we constructed the general odd solution and the marginal condition between $S$ and $q_{c}$ (Fig. 1). Clearly, the even solution is encountered first as $S$ increases from zero. Hence this even solution governs the onset of convection.

Whereas descending fronts are stable against any small perturbations, ascending fronts are unstable to longwavelength perturbations due to the buoyancy of the lighter fluid below the front. Accordingly, for any specified value of the driving parameter $S$ in Fig. 1, wave numbers satisfying $q<q_{c}$ are unstable to convection. The physical source of this instability is embodied in the driving parameter $S$, which measures the strength of the destabilizing influence of buoyancy relative to the stabilizing influence of curvature. For a given initial perturbation amplitude, the smaller the perturbation wavelength, the larger the curvature. Curvature effects therefore stabilize small-wavelength perturbations.

The unique even solution $A=C, A_{n}=C_{n}$, and $A(\gamma)=C(\gamma)$ guarantees that the velocity is vertical at the front. This is also true for the odd solution. These solutions correspond to two different types of fluid flow. For the even solution, $v_{e}$ and $w_{e}$ are symmetric about the $x$ axis. Furthermore, numerical results show that $u_{e}$ is very small for reasonably small $q_{c}\left(\left|u_{e}\right| \approx 0.005\left|w_{e}\right|\right.$ for $q_{c}=1.0$ ) and that the corresponding convection pattern is composed of a roll structure of wave number $q_{c}$ in the $y-z$ plane parallel to the slab (see Fig. 2). For the odd solution, the projection of the pattern on the $x-z$ plane perpendicular to the slab is made up of a roll structure

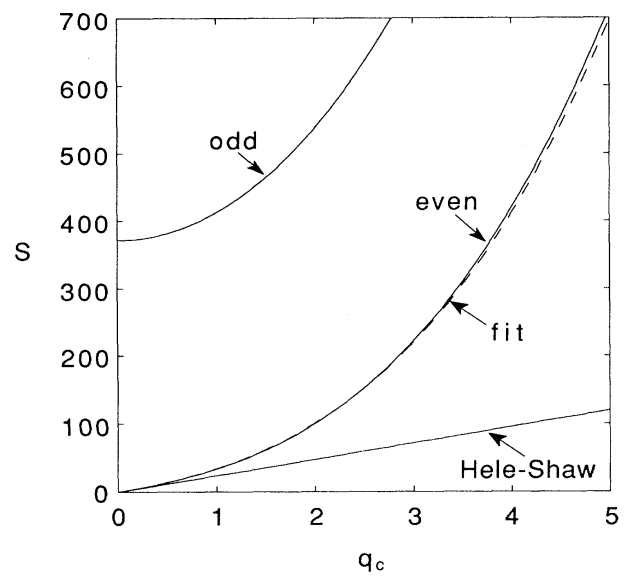

FIG. 1. Convective stability diagram for flat ascending reaction fronts in a vertical slab. Marginal stability traces are shown for even and odd flows about the midplane parallel to the slab. The exact analytical Hele-Shaw $(S \rightarrow 0)$ limit $S=24 q_{c}$ for even flow is shown. The dashed trace is from an approximate analytical form, Eq. (40). For a particular value of $S$, the band $0<q<q_{c}$ of perturbation wave numbers is unstable to convection.

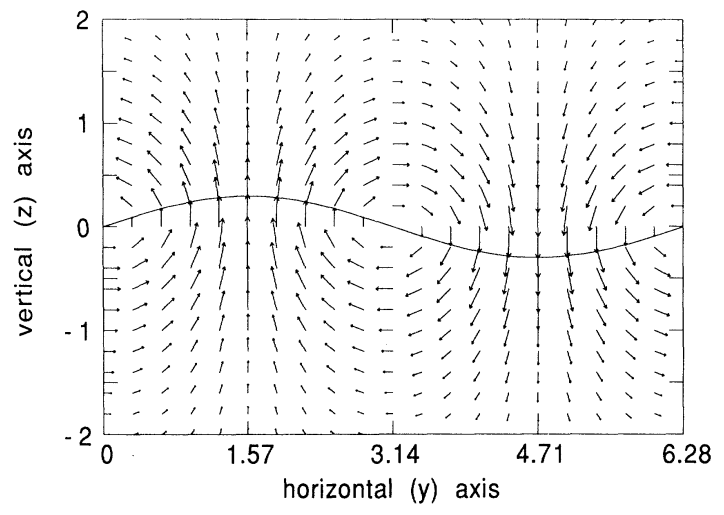

FIG. 2. Velocity field at the onset of convection for perturbation of wave number $q=1.0$ about a flat ascending horizontal reaction front in a vertical slab, showing that the vertical extent of the flow pattern is comparable to one horizontal wavelength. The sinusoidal trace represents the position of the perturbed front.

whose wavelength is fixed by the slab width $a$, and the projection on the $y-z$ plane is also made up of a roll structure of wave number $q_{c}$. Thus, the combined pattern for the odd solution is rather complicated.

The limit of $q \rightarrow 0$ reduces the odd solution to twodimensional flow in the $x-z$ plane perpendicular to the slab. A previous calculation using a similar technique [6] in two dimensions yielded $S_{c}=371.5$ for this flow. This is in good agreement with our $q \rightarrow 0$ limit $S=371$. 1 .

\section{HELE-SHAW CELL $(S \rightarrow 0$ limit $)$}

Simple instructive results can be obtained in the $S \rightarrow 0$ limit, where the system is effectively two-dimensional. One way to have small $S=\delta g a^{3} / v D_{C}$ is to have a narrow "Hele-Shaw" slab [10] with small $a$. In the $S \rightarrow 0$ limit, the Navier-Stokes equations are replaced by Darcy's law [11],

$$
\begin{aligned}
& \mathbf{v}(y, z)=-\frac{a^{2}}{12 \mu} \nabla_{\|}(p+\rho g z), \\
& \boldsymbol{\nabla}_{\|} \cdot \mathbf{v}=0,
\end{aligned}
$$

where $\nabla_{\|}=(0, \partial / \partial y, \partial / \partial z)$ is the gradient parallel to the slab. Substituting Eq. (30) into (31) yields the Laplace equation. In our dimensionless units, we can write this equation as

$$
\boldsymbol{\nabla}_{\|}^{2} \boldsymbol{\Phi}=0,
$$

where $\Phi=\left(P+a^{3} \rho g v^{-2} z\right) / 12$.

We also require Eq. (2c),

$$
\widehat{\mathbf{n}} \cdot\left[\widehat{\mathbf{z}} \frac{\partial h}{\partial t}-\left.\mathbf{v}\right|_{z=h}\right]=v_{0}+\frac{D_{C}}{v} \kappa
$$

and the jump condition $[P]_{-}^{+}=0$, which can be written as

$$
[\Phi]_{-}^{+}=-\frac{D_{C} S}{12 v} h .
$$


The normal component of velocity is continuous across the front [11]. To study the onset of convection, we introduce small perturbations about the flat front as mentioned in Sec. II. Linearizing in the perturbations yields

$$
\begin{aligned}
& \nabla_{\|}^{2} \Phi^{(1)}=0, \\
& \frac{\partial h^{(1)}}{\partial t}=\frac{D_{C}}{v} \frac{\partial^{2} h^{(1)}}{\partial y^{2}}+\left.\frac{\partial \Phi^{(1)}}{\partial z}\right|_{z=0}, \\
& {\left[\Phi^{(1)}\right]_{-}^{+}=-\frac{D_{C} S}{12 v} h^{(1)} .}
\end{aligned}
$$

After writing the perturbed front position as

$$
h^{(1)}=E e^{i q y+\sigma t}
$$

we find the solution of Eqs. (33a) and (33b),

$$
\begin{aligned}
& \Phi^{(1)}(y, z, t)=-\frac{E}{q}\left(\sigma+D_{C} q^{2} / v\right) e^{i q y-q z+\sigma t} \quad(z \geq 0), \\
& \Phi^{(1)}(y, z, t)=\frac{E}{q}\left(\sigma+D_{C} q^{2} / v\right) e^{i q y+q z+\sigma t} \quad(z<0) .
\end{aligned}
$$

Finally, the jump condition Eq. (33c) yields a telling analytical result for the growth rate;

$$
\sigma=\frac{D_{C}}{v} q\left[\frac{S}{24}-q\right] \text {. }
$$

Accordingly, the flat front is unstable $(\sigma>0)$ for perturbations with wave number $q$ satisfying $S>24 q$. The cutoff wave number $q_{c}$ is given by the marginal condition

$$
S=24 q_{c}
$$

for the vanishing growth rate, which agrees well with the $S \rightarrow 0$ limit of the even solution (see Fig. 1). Thus, the Hele-Shaw cell is indeed a good approximation for small driving parameters $S$. By setting the derivative of Eq. (36) with respect to $q$ equal to zero, we obtain the wave number $q_{m}=q_{c} / 2$ of maximum growth rate $\sigma_{m}=D_{C} S^{2} / 48 v$. For the laterally unbounded system, the ratio $q_{m} / q_{c}$ depends on the molecular diffusivity and the viscosity [4], whereas here for a vertical slab with small $S$, the maximum growth rate occurs at exactly half the cutoff wave number.

\section{DISCUSSION AND IMPLICATIONS FOR EXPERIMENTS}

The fully three-dimensional linear stability analysis of ascending autocatalytic reaction fronts in vertical slabs shows instability for large-wavelength perturbations at all finite values of the dimensionless driving parameter $S$. This instability is driven by buoyancy, which dominates over the competing curvature dependence of the front velocity in a band $0<q<q_{c}$ of unstable dimensionless wave numbers. The cutoff wave number $q_{c}$ is determined only by $S$ according to Fig. 1, and should be observable in experiments.

To date, the theory of convection in autocatalytic reaction fronts has been compared only with experiments on vertical cylinders [6]. Although these comparisons are favorable, the experimental uncertainties are so large as to render the comparisons only semiquantitative; the experimental critical diameter for onset of convection is known only to within a factor of two. Thus, careful experimental tests of the linear theory, either in cylinders or in the slab geometry discussed in this paper, are needed.

Experiments designed to test the predicted cutoff wave number $q_{c}$ as a function of the driving parameter $S=\delta g a^{3} / v D_{C}$ might be carried out by varying any of the parameters in $S$, such as the density jump $\delta$ (through the various chemical concentrations) or the slab width $a$. To introduce perturbations of a specified wavelength $\lambda$ measured in conventional units, the chemical reaction could be initiated (a) at an electrode whose top horizontal edge has a sinusoidal shape or (b) at the bottom of the slab simultaneously at several points separated by this wavelength. If $\lambda<\lambda_{c}=2 \pi a / q_{c}$, where $q_{c}$ is taken from Fig. 1 based on the value of $S$, the perturbations should decay with time, whereas if $\lambda>\lambda_{c}$, they should grow with time. For example, Fig. 3 shows $\lambda_{c}$ for the even solution in Fig. 1 as a function of the slab width $a$ for the typical values $[1,2] \delta=0.90 \times 10^{-4}, \dot{g}=980 \mathrm{~cm} / \mathrm{sec}^{2}, \quad v=9.2 \times 10^{-3}$ $\mathrm{cm}^{2} / \mathrm{sec}$, and $D_{C}=2.0 \times 10^{-5} \mathrm{~cm}^{2} / \mathrm{sec}$; the Hele-Shaw result $\lambda_{c}=48 \pi v D_{C} / \delta g a^{2}$ obtained from Eq. (37) is also shown. The solid trace divides the phase space of perturbation wavelengths $\lambda$ and slab widths $a$ into two regions; the region including (excluding) the origin is stable (unstable) to convection. Note that whereas Fig. 1 is universal, the details of Fig. 3 depend on the type of the reaction, the initial chemical concentrations, the fluid viscosity, etc.

The cutoff wavelength $\lambda_{c}$ in Fig. 3 seems to approach a constant limiting value as $a \rightarrow \infty$, the limit of large slab widths. We now show that this limiting value is simply the cutoff wavelength for a laterally unbounded slab, as might be expected. After rewriting in the present units, a relation between the cutoff wave number and the driving parameter for a laterally unbounded slab [Eq. (40) of Ref. [4] ] reads as

$$
S=4 q_{c}^{3} \text {. }
$$

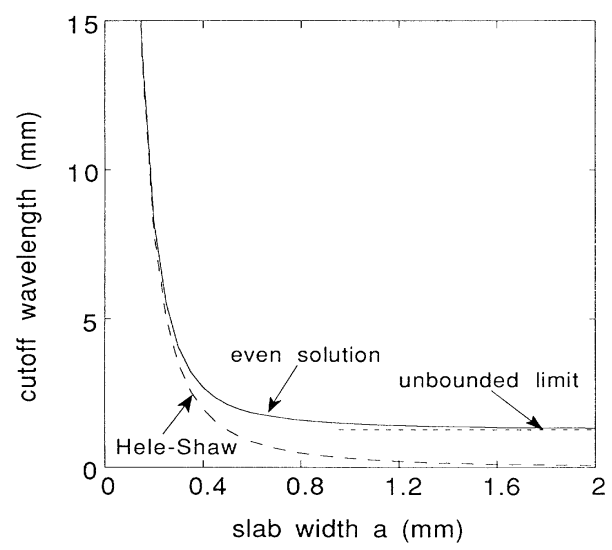

FIG. 3. Cutoff wavelength $\lambda_{c}$ versus slab width $a$ for the iodate-arsenous acid system (solid trace), including the HeleShaw and laterally unbounded limits. 
This result implies a relation for the cutoff wavelength

$$
\lambda_{c}=2 \pi\left(\frac{4 v D_{C}}{g \delta}\right)^{1 / 3}
$$

which is manifestly independent of $a$. This relation gives $\lambda_{c}=1.27 \mathrm{~mm}$ (dotted horizontal line, Fig. 3) for the fluid parameters specified above; this value evidently agrees well with the even solution in the limit $a \rightarrow \infty$.

An approximate analytical marginal condition relating $S$ and $q_{c}$ may prove useful when preparing plots like Fig. 3 for different experimental situations. To construct such a condition, we modify Eq. (26), which already satisfies the $S \rightarrow \infty\left(q_{c} \rightarrow \infty\right)$ limit [Eq. (38)], to enforce agreement with the $S \rightarrow 0\left(q_{c} \rightarrow 0\right)$ limit [Eq. (37)]. This is done simply by replacing the first appearance of $\pi^{2}$ in Eq. (26) with the number 12 , yielding

$$
S=2 q_{c}\left(12+q_{c}^{2}+q_{c} \sqrt{18+q_{c}^{2}}\right) .
$$

We have also replaced the second appearance of $\pi^{2}$ with the number 18 to obtain the best overall fit to the even solution data, resulting in differences between the approximate and exact conditions within $2.5 \%$ over the whole range of $S$. The corresponding fit shown in Fig. 1 is for small $q_{c}$ only; the maximum difference occurs at $q_{c} \approx 6$.

Experiments designed to carefully test the present theory should involve (1) slab widths $a$ and perturbation wavelengths $\lambda$ that are small compared with the long horizontal dimension $l$ of the experimental slab, (2) perturbations which are sinusoidal and of small amplitude, and (3) perturbed fronts which are several wavelengths away from the bottom of the slab when their stability is being analyzed. These requirements might be realized by using a wedged slab whose width $a$ varies slowly with height in such a way that the marginal-stability height, where $\lambda=\lambda_{c}(a)$, would occur a few wavelengths or more above the bottom. Such a geometry would allow the perturbation amplitude to decay with time (while the perturbation wavelength remains constant) until the front reaches the marginal stability height, where the perturbation would be of small amplitude and of approximately sinusoidal shape. A single experimental apparatus may be sufficient to test a range of experimental wavelengths, especially if the chemical concentrations (and hence $\delta$ ) are varied. The variation of $a$ with height should be slow enough to be considered constant over a vertical distance of one wavelength.

For a nonsinusoidal but still periodic perturbation, higher spatial harmonics would have smaller wavelengths $\lambda_{n} \equiv \lambda / n$ than the fundamental wavelength $\lambda$, where $n=2,3, \ldots$. Figure 3 shows that these higher harmonics will not become unstable before the fundamental wavelength itself becomes unstable, consistent with the idea that velocity effects stabilize short wavelengths. Thus, a sinusoidal perturbation may not be essential. Undesired perturbations with wavelengths larger than the desired perturbation wavelength, which generally render the perturbation nonperiodic, are ubiquitous in experiments even though care in preparing the initial state can ensure that their amplitudes are small compared with the ampli- tude of the desired perturbation. The growth rates of such undesired wavelengths will typically exceed those of the desired perturbation wavelengths near the cutoff. Whether or not the amplitudes of these undesired wavelengths become appreciable, it may be useful to record the full evolution of the entire front to allow a spectral analysis of the wavelength-dependent growth rate. Two properties of iodate-arsenous acid reaction fronts are helpful here. First, the front is observable optically (and visually), being the interface between two different colors of unreacted fluid and of reacted fluid. Second, typical front propagation speeds $c_{0} \approx 3 \times 10^{-3} \mathrm{~cm} / \mathrm{sec}$ are very slow because of the slow molecular diffusion of the catalyst, leaving time even to scan the front surface before it moves appreciably.

In experiments, fluids must be bounded in both horizontal directions. If the horizontal dimensions of the slab are $a$ and $l>a$, the maximum possible perturbation wavelength is $2 l$ which corresponds to one roll in the cell. Thus, wavelengths $\lambda>2 l$ and dimensionless wave numbers $q<q_{\min }=\pi a / l$ are inaccessible experimentally. Consequently, finite slabs have a nonzero critical driving parameter for onset of convection given approximately by $S_{c}=24 q_{\min }=24 \pi a / l$, below which such slabs are stable to convective perturbations of all wavelengths. Even though using the $S \rightarrow 0$ limit and ignoring the boundary conditions at the narrow sidewalls render this critical driving parameter approximate, we nevertheless expect such boundary-induced criticality to survive in calculations in which these assumptions are relaxed.

In previous experiments on this system, a reaction was initiated at one end of a vertical tube and allowed to propagate through the tube, consuming the reactants. Such experiments were necessarily time-limited; also, they did not allow convenient tuning of experimental parameters. However, this kind of experiment can be turned into a steady state, tunable system by continually flowing fresh reactant solution down the cell from above, and by making the cell slightly tapered. In a vertical slab with a wedge opening downward, i.e., so that the width decreases with height, this flow has a stable steady state, as long as one accepts the plausible assumption that the convective velocity $v_{c}$ of the front relative to the fluid is an increasing function $v_{c}(S)$ of the control parameter $S$. In this case, the reaction front will move to the stable vertical position where the local control parameter is such that $v_{c}(S)$ matches the local average flow velocity $v_{f}$. Changing the externally imposed flow rate will cause the front to move to a different vertical level, allowing observations to be made at a different value of $S$. Apart from the pattern-forming instability discussed in this paper, this equilibrium is stable, whereas the opposite case, in which the wedge opens upwards, in unstable. A front that is momentarily below its stable equilibrium position, for example, experiences higher $S$ and lower $v_{f}$ in a downward-opening wedge; these effects combine to restore the front to its equilibrium position. In preliminary experiments of this type, a vertically translatable horizontal electrode has allowed us to initiate the reaction at any desired vertical position in the wedge. These experiments have not yet yielded useful results on the stability of the 
resulting reaction front.

Intriguing questions remain about the fate of autocatalytic reaction fronts carried into the nonlinear regime. Above criticality $\left(S>S_{c}\right)$, the system must choose from a band $q_{\min }<q<q_{c}$ of unstable wave numbers. If a particular wavelength is chosen by the initial conditions or if a band of wavelengths is chosen by the fluid itself in a vertical slab (a) will the front eventually reach a steady saturated periodic shape in which all points on the front propagate with the same constant vertical speed, (b) will stable or unstable fingers appear, or (c) will secondary instabilities drive the system to some more favorable wavelength such as, perhaps, the rather uninteresting maximum wavelength $l$ allowed by the boundaries? Will the fluid invert itself catastrophically, so that the lighter reacted fluid eventually finds itself above the unreacted fluid, and the system is absolutely unstable? If the answer to the last question is yes, are there other geometries or flow configurations that might allow a steady saturated periodic front shape and flow pattern?

If steady periodic fronts can be realized in autocatalytic systems, many interesting questions about pattern formation will emerge. What is the preferred wavelength as a function of the driving parameter $S$ ? When would the steady pattern become unstable and what secondary instability would destabilize it? What is the route to chaos in such a system?

In conclusion, limited understanding of the patternforming behavior of autocatalytic reaction fronts has prompted the present calculation of linear stability for a vertical slab, which identifies the basis instability. However, many questions remain regarding the flows resulting from this basic instability. To answer these questions requires further investigation.

\section{ACKNOWLEDGMENTS}

We are indebted to Joseph W. Wilder for many insightful discussions of this work and for carefully reading the manuscript. The preliminary experiments referred to in this paper were performed by one of us (P.K.) in collaboration with Sibel Bayrakci. Support from National Science Foundation Grant No. RII-8922106 and the National Research Center for Coal and Energy are gratefully acknowledged.
[1] T. McManus, Ph.D. thesis, West Virginia University, 1989, Chap. 3.

[2] J. A. Pojman, I. R. Epstein, T. J. McManus, and K. Showalter, J. Phys. Chem. 95, 1299 (1991).

[3] A. Saul and K. Showalter, in Oscillations and Traveling Waves in Chemical System, edited by R. J. Field and M. Burger (Wiley, New York, 1985), p. 419.

[4] B. F. Edwards, J. W. Wilder, and K. Showalter, Phys. Rev. A 43, 749 (1991).

[5] J. P. Keener and J. J. Tyson, Physica D 32, 307 (1988); J. W. Wilder, D. A. Vasquez, and B. F. Edwards, Phys. Rev. E 47, 3761 (1993).

[6] D. A. Vasquez, B. F. Edwards, and J. W. Wilder, Phys.
Rev. A 43, 6694 (1991); D. A. Vasquez, J. W. Wilder, and B. F. Edwards, Phys. Fluids A 4, (11), 2410 (1992).

[7] J. W. Wilder, B. F. Edwards, and D. A. Vasquez, Phys. Rev. A 45, 2320 (1992); D. A. Vasquez, B. F. Edwards, and $\mathbf{J}$. W. Wilder (unpublished).

[8] D. A. Vasquez, J. W. Wilder, and B. F. Edwards, J. Chem. Phys. 98, 2138 (1993).

[9] J. Boussinesq, Théorie Analytique de la Chaleur 2 (Gauthier-Villars, Paris, 1903); A. Oberbeck, Ann. Phys. Chem. (Leipzig) 7, 271 (1879).

[10] H. S. S. Hele-Shaw, Nature (London) 58, 34 (1898).

[11] P. G. Saffman and G. I. Taylor, Proc. R. Soc. London Ser. A 245, 312 (1958). 